\title{
Blood Pressure and Transient Postoperative Neurologic Deterioration, Following Superficial Temporal-to-middle Cerebral Artery Anastomosis in Adult Patients With Moyamoya Disease
}

\section{Tak Kyu Oh}

Seoul National University Bundang Hospital

Ji Hyeon Kim

Seoul National University Bundang Hospital Ho-Young Lee

Seoul National University Bundang Hospital

\section{Seong-Eun Kim}

Seoul National University Bundang Hospital

\section{Tackeun Kim}

Seoul National University Bundang Hospital

Beom Joon Kim

Seoul National University Bundang Hospital

Jae Seung Bang

Seoul National University Bundang Hospital

\section{Moon-Ku Han}

Seoul National University Bundang Hospital

\section{Chang Wan Oh}

Seoul National University Bundang Hospital

Hee-Joon Bae ( $\nabla$ braindocbae@gmail.com )

Seoul National University Bundang Hospital

Young-Tae Jeon ( $\nabla$ ytjeonsnubh@gmail.com )

Seoul National University Bundang Hospital

\section{Research article}

Keywords: Neurosurgery, Blood Pressure, Neurologic Manifestations, General Surgery, Postoperative Complications

Posted Date: August 1st, 2020 
DOI: https://doi.org/10.21203/rs.3.rs-46105/v1

License: (c) (1) This work is licensed under a Creative Commons Attribution 4.0 International License. Read Full License 


\section{Abstract \\ Background}

This study investigated whether intraoperative systolic blood pressure (ISBP) is associated with the risk of transient neurologic deficits (TND), following superficial temporal-to-middle cerebral artery (STA-MCA) anastomosis in adult patients with moyamoya disease (MMD).

\section{Methods}

In this retrospective observational study, data from adult patients with MMD who had undergone MCASTA anastomosis at a single tertiary academic hospital during May 2003-April 2014 were examined. ISBP data were extracted from our clinical data warehouse. Data on patient characteristics were obtained from electronic medical records, including the details of comorbidities and laboratory findings. TND was the primary outcome of interest.

\section{Results}

Out of 192 patients (228 hemispheres), 66 (29\%) hemispheres had TND after surgery. There were significant differences in ISBP between patients with and without TND. The lowest ISBP quartile was independently associated with TND (odds ratio: 5.50; 95\% confidence interval: 1.96-15.46).

\section{Conclusions}

Low ISBP might lead to TND after STA-MCA anastomosis in adult patients with MMD. In patients with poor perfusion status, low ISBP was associated with an increased risk of TND. Our findings suggest that strict ISBP control might be required to prevent TND after anastomosis in patients with MMD, in particular, patients with poor perfusion status.

\section{Background}

Superficial temporal-to-middle cerebral artery (STA-MCA) anastomosis is a surgical treatment to reduce the risk of cerebral ischemia in patients with moyamoya disease (MMD).[1] Transient neurological deficits (TND) can occur due to cerebral hyper-[2-4] or hypoperfusion[5] associated with this procedure. Previous studies have reported several risk factors for TND, including adult-onset,[6] haemorrhagic-onset, [6] poor vascular response before surgery,[4] surgery to the dominant hemisphere,[7] and increased postoperative white blood cell counts.[7]

Blood pressure (BP) variability has been associated with neurologic deterioration, following acute ischemic stroke.[8] A common practice in MMD management is to keep BP at or just above the 
preoperative baseline.[9] A decline in the mean arterial pressure during surgery has been reported to cause cerebral ischemia by decreasing cerebral perfusion pressure and cerebral blood flow in childhood MMD patients, receiving revascularization surgery.[10] In adult MMD patients undergoing STA-MCA anastomosis, associations between perioperative cerebral perfusion status and post-operative outcomes have been suggested.[11] However, to the best of our knowledge, there has been no previous study to examine whether perioperative BP affects the risk of TND in these patients. The aim of the present study was to elucidate whether intraoperative BP status is associated with the risk of TND, following STA-MCA anastomosis in adult patients with MMD, and whether pre-operative cerebral perfusion status might mediate this association.

\section{Methods}

\subsection{Design and ethical statement}

This retrospective observational study was approved by the institutional review board of Seoul National University Bundang Hospital (IRB No.B-1411/274 -113). The requirement for patient consent was waived due to the retrospective nature of this study and minimal risk to study participants. Data were extracted from electronic medical records.

\subsection{Study subjects and data collection}

We collected data on patients with adult MMD who had undergone MCA-STA anastomosis at Seoul National University Bundang Hospital between May 2003 and April 2014. Two hundred forty-eight hemispheres of 208 consecutive patients met the inclusion criteria; however, 20 hemispheres were subsequently excluded due to insufficient data (8), postoperative cerebral haemorrhage (6), and postoperative cerebral infarction (6), leaving 228 hemispheres in the final sample.

Patient data were extracted from electronic medical records, including patient characteristics, details of comorbidities, and laboratory findings. BP data were collected through routine clinical practice and downloaded from our institution's clinical data warehouse for the purpose of this study.

\subsection{Surgical and anaesthetic procedures}

General indication for revascularization surgery included apparent cerebral ischemia or decreased regional blood flow, vascular response, and reserve in perfusion studies in our hospital.[12] Digital subtraction angiography was performed to diagnose MMD, evaluating collateral channels and identifying appropriate donor and recipient. Thereafter, perfusion status was evaluated with computed tomography (CT) or single-photon emission computed tomography (SPECT) with acetazolamide challenge, useful for evaluating resting perfusion and reserve capacity.

Regarding anaesthetic techniques, similar protocols were followed with study participants. Radial artery cannulation was performed to perioperatively monitor blood pressure. Total intravenous anaesthesia with propofol and remifentanil was administered with a target-controlled infusion pump. Intraoperative 
systolic BP (ISBP) was maintained above the level of the preoperative baseline value. Normocapnia and normovolemia were maintained.

After surgery, brain CT was performed to detect any postoperative infarction or haemorrhage. Patients were admitted to a neurological intensive care unit. Neurological examination was performed once patients regained consciousness. SBP was kept within $20 \mathrm{mmHg}$ of the preoperative level. Patients' volume status was maintained as euvolemic. If neurological symptoms developed after surgery, SPECT or perfusion CT was performed to evaluate changes to the cerebral perfusion status.

\subsection{Brain Perfusion Diamox SPECT image analysis}

Two nuclear medicine physicians (HYL, JHK) performed independent visual assessments of the SPECT images of each cerebral hemisphere. Disagreements were resolved by discussion and consensus between the physicians. Perfusion status detected on brain SPECT images was classified as "normal", "mild decreased", "moderate decreased", "severe decreased", or "defect". Decreased perfusion was defined as cerebral perfusion on basal SPECT within a lower colour range compared to contralateral side and/or the surrounding area. Decreased cerebrovascular reserve (CVR) was defined as abnormal cerebral perfusion within a lower colour range relative to basal SPECT, after acetazolamide administration. The severity of decreased CVR was classified into "mild", "moderate", "severe", and "defect".[13] We evaluated the concordance rate between the physicians' evaluation, which was $89.5 \%$.

\subsection{Outcome assessment}

The primary outcome of interest was the occurrence of TND after surgery. Based on a previous report,[3] TND was confirmed if the patient met all of the following criteria: (1) presence of neurological deficits that did not exist before surgery; (2) TND resolved completely within 15 days of operation; (3) presence of a significant focal increase or decrease in cerebral blood flow at the anastomosis site, captured by a postoperative SPECT scan; and (4) no hematoma or acute infarction detected on brain CT or diffusionweighted magnetic resonance imaging.

\subsection{Statistical analysis}

ISBP data were summarized as a mean, categorized into four groups, using quartiles, and examined for an association with potentially confounding variables. One-way ANOVA or Kruskal-Wallis test were conducted for continuous variables, and chi-square test or Fisher's exact test was conducted for categorical variables.

According to TND, the categorized mean ISBP was compared using the chi-square test or Fisher's exact test. Proportion of confirmed TND cases per mean ISBP category was plotted against the patients' cerebral perfusion status. The association between TND and mean ISBP was examined by comparing odds ratios (ORs) in a logistic regression model. ORs were adjusted for confounding variables in a multivariable model. Clinically meaningful variables as well as the variables with $P$-value less than 0.1 in the univariate analysis were for the model. The following variables were included in the multivariable model: age, cerebral perfusion status, operation duration, operation site, history of stroke, duration of 
hospitalization, pre-operation mean SBP, preoperative haemoglobin level, and post-operative haemoglobin level.

In the multivariable logistic regression model, a non-linear term representing the mean ISBP was included to examine a non-linear relationship between mean ISBP and TND risk. To control for multi-collinearity, cantered mean ISBP was used. Moreover, cerebral perfusion status was included as an interaction term to verify its impact on the association between mean ISBP and TND risk.

Finally, the proportion of confirmed TND cases per ISBP category predicted by the multivariable regression model were plotted per mean ISBP according to the cerebral perfusion status, controlling for relevant confounders, whereby averages and reference values were used for continuous and categorical variables, respectively. Statistical analyses were performed using SAS 9.4 (SAS Institute Inc., Cary, NC). Pvalues $<0.05$ were considered statistically significant.

\section{Results}

Among 228 hemispheres of 192 patients, 66 hemispheres (29\%) had confirmed TND after surgery. Among these patients, 30,853 ISBP measurements were taken, with an average of 136 measurements per patient. Mean (standard deviation, SD) of ISBP per patient was 129.6 (10.9) and 14.8 (4.0). There were no significant differences in baseline characteristics among the groups based on ISBP quartiles (Table 1). TND was significantly associated with mean ISBP $(p=0.005$, Table 2$)$. Using ISBP values within 125$133 \mathrm{mmHg}$ (second and third quartile, respectively) as a reference, the odds of TND in patients with mean ISBP < $120.2 \mathrm{mmHg}$ were $5-6$ times greater than in the reference group (crude OR $=4.78,95 \% \mathrm{Cl}$ : $1.92-$ 11.91 ; adjusted $\mathrm{OR}=5.50,95 \% \mathrm{Cl} 1.96-15.46$ ). Meanwhile, for patients with mean ISBP in the 125$133 \mathrm{mmHg}$ range, the odds of TND were 3 times greater than for the reference group (crude $\mathrm{OR}=2.83$, 95\% Cl: 1.11-7.19; adjusted OR =3.24, 95\% Cl: 1.16-9.05) (Table 2). 
Table 1

Comparison of baseline characteristics by SBP mean quartiles

\begin{tabular}{|c|c|c|c|c|c|}
\hline Variable & $\begin{array}{l}<\mathrm{Q1}(< \\
120.2) \\
(\mathrm{N}=57)\end{array}$ & $\begin{array}{l}\mathrm{Q} 1-\mathrm{Q} 2 \\
(120.2-125) \\
(\mathrm{N}=57)\end{array}$ & $\begin{array}{l}\text { Q2 - Q3 (125- } \\
133) \\
(\mathrm{N}=57)\end{array}$ & $\begin{array}{l}\geq \mathrm{Q4}(\geq \\
133) \\
(\mathrm{N}=57)\end{array}$ & $\begin{array}{l}\mathrm{P}- \\
\text { value* }\end{array}$ \\
\hline Age (year) & $38.9(13.2)$ & $39.0(12.4)$ & $41.5(12.0)$ & $41.7(12.2)$ & 0.471 \\
\hline Gender: Male & $20(23)$ & $25(29)$ & $17(20)$ & $24(28)$ & 0.382 \\
\hline $\mathrm{BMI}\left(\mathrm{kg} / \mathrm{m}^{2}\right)$ & $25.2(4.2)$ & $24.8(4.0)$ & $26.6(4.3)$ & $26(4.3)$ & 0.129 \\
\hline \multicolumn{6}{|l|}{ Comorbidities } \\
\hline Previous Stroke & $9(32)$ & $5(18)$ & $6(21)$ & $8(29)$ & 0.653 \\
\hline Hypertension & $16(21)$ & $17(23)$ & $22(29)$ & $20(27)$ & 0.613 \\
\hline Diabete Mellitus & $6(18)$ & $12(35)$ & $9(26)$ & $7(21)$ & 0.407 \\
\hline Smoking & $13(27)$ & $16(33)$ & $8(16)$ & $12(24)$ & 0.333 \\
\hline $\begin{array}{l}\text { Coronary Artery } \\
\text { Disease }\end{array}$ & 0 & 0 & $2(100)$ & 0 & $0.247 \S$ \\
\hline Thyroid Disease & $4(29)$ & $4(29)$ & $3(21)$ & $3(21)$ & $1.000 \S$ \\
\hline \multicolumn{6}{|l|}{$\begin{array}{l}\text { Clinical } \\
\text { Characteristics }\end{array}$} \\
\hline Anesthesia Time (min) & $479(91)$ & $474(100)$ & $499(102)$ & $492(95)$ & 0.487 \\
\hline Operation Time (min) & $408(86)$ & $406(108)$ & $423(99)$ & $424(93)$ & 0.642 \\
\hline Preoperative Hb (g/dl) & $13.8(1.6)$ & $13.8(1.7)$ & $13.5(1.6)$ & $13.5(1.6)$ & 0.451 \\
\hline $\begin{array}{l}\text { Preoperative WBC } \\
\left(10^{3} / \mathrm{ul}\right)\end{array}$ & $7.0(1.9)$ & $7.0(1.7)$ & $6.7(1.4)$ & $6.7(1.5)$ & 0.477 \\
\hline $\begin{array}{l}\text { Length of Hospital } \\
\text { Stay (day) }\end{array}$ & $13.4(4.7)$ & $12.5(4.4)$ & $12.4(4.4)$ & $14.8(7.5)$ & 0.07 \\
\hline $\begin{array}{l}\text { Operation site } \\
\text { (Right/Left) }\end{array}$ & $\begin{array}{l}31(24) / 26 \\
(27)\end{array}$ & $\begin{array}{l}33(25) / 24 \\
(24)\end{array}$ & $\begin{array}{l}34(26) / 23 \\
(23)\end{array}$ & $\begin{array}{l}32(25) / 25 \\
(26)\end{array}$ & 0.949 \\
\hline $\begin{array}{l}\text { Cerebral Perfusion } \\
\text { status }\end{array}$ & $8(30.8)$ & $5(19.2)$ & $4(15.4)$ & $9(34.6)$ & 0.399 \\
\hline \multicolumn{6}{|c|}{ Values are expressed as the mean (standard deviation) or number (percentage, \%) } \\
\hline \multicolumn{6}{|c|}{ *By One-Way ANOVA or Chi-square test as appropriate } \\
\hline \multicolumn{6}{|c|}{ §By Kruskal-Wallis test or Fisher's exact test as appropriate } \\
\hline SBP, Systolic Blood Pre & ure; BMI, Bod & ass Index; $\mathrm{HI}$ & ylok & Xto h hord ol & \\
\hline
\end{tabular}


Table 2

Occurrence of TND according to ISBP quartiles

\begin{tabular}{|c|c|c|c|c|c|}
\hline ISBP Quartiles & $\begin{array}{l}\text { TND (+) } \\
(N=66)\end{array}$ & $\begin{array}{l}\text { TND }(-) \\
(N=162)\end{array}$ & $\begin{array}{l}P \\
\text { value* }\end{array}$ & $\begin{array}{l}\text { Crude OR }(95 \% \\
\text { Cl)§ }\end{array}$ & $\begin{array}{l}\text { Adjusted OR (95\% } \\
\text { Cl) }+\end{array}$ \\
\hline$<$ Q1 $(<120.2)$ & $\begin{array}{l}25 \\
(43.9 \%)\end{array}$ & $\begin{array}{l}32 \\
(56.1 \%)\end{array}$ & 0.005 & $4.78(1.92,11.91)$ & $5.50(1.96,15.46)$ \\
\hline $\begin{array}{l}\text { Q1 - Q2 (120.2- } \\
125)\end{array}$ & $\begin{array}{l}18 \\
(31.6 \%)\end{array}$ & $\begin{array}{l}39 \\
(68.4 \%)\end{array}$ & & $2.83(1.11,7.19)$ & $3.24(1.16,9.05)$ \\
\hline Q2 - Q3 (125-133) & $8(14.0 \%)$ & $\begin{array}{l}49 \\
(86.0 \%)\end{array}$ & & reference & reference \\
\hline$\geq \mathrm{Q} 3(\geq 133)$ & $\begin{array}{l}15 \\
(26.3 \%)\end{array}$ & $\begin{array}{l}42 \\
(73.7 \%)\end{array}$ & & $2.19(0.84,5.67)$ & $1.39(0.48,4.04)$ \\
\hline \multicolumn{6}{|c|}{ *By Chi-square test as appropriate or Fisher's exact test as appropriate } \\
\hline \multicolumn{6}{|c|}{$\S B y$ logistic regression model; modeling the probability of TND } \\
\hline \multicolumn{6}{|c|}{$\begin{array}{l}\text { †By logistic regression model; modeling the probability of TND adjusted for age, operation time, } \\
\text { operation site, cerebral perfusion status, history of stroke, duration of hospitalization, pre operation } \\
\text { SBP mean, preoperative hemoglobin, and postoperative hemoglobin. }\end{array}$} \\
\hline
\end{tabular}

The impact of mean ISBP on TND risk was mediated by the cerebral perfusion status Fig. 1). However, the quadratic term and interaction test result were non-significant $(p=0.355$ and $p=0.157$, respectively).The mean ISBP had an effect on the proportion of TND cases ( $P$-value $=0.026)$, which was independent of the patients' cerebral perfusion status $(P$-value $=0.123)$. The risk of TND decreased as the mean ISBP increased. In addition, pre-operative mean SBP, operation site, pre-operative haemoglobin level, and hospital stay duration were associated with the proportion of TND cases confirmed within each mean ISBP category. Overall, in each mean ISBP group, patients with a higher mean pre-operative SBP or pre-operative haemoglobin level, longer hospital stay, and patients operated on the left side were more likely to develop TND (Table 3). 
Table 3

Estimates from the multivariable logistic regression models on ISBP mean

\begin{tabular}{|lcccccc|}
\hline Model & Linear & \multicolumn{3}{c}{$\begin{array}{l}\text { Nonlinear } \\
\text { without } \\
\text { interaction }\end{array}$} & \multicolumn{3}{c|}{$\begin{array}{l}\text { Nonlinear } \\
\text { with interaction }\end{array}$} \\
\hline Parameter & Estimate & $\begin{array}{l}\text { P- } \\
\text { value }\end{array}$ & Estimate & $\begin{array}{l}\text { P- } \\
\text { value }\end{array}$ & Estimate & $\begin{array}{l}\text { P- } \\
\text { value }\end{array}$ \\
\hline ISBP mean & -0.0883 & 0.026 & - & - & - & - \\
\hline Cerebral perfusion status & 7.3463 & 0.124 & -0.0558 & 0.833 & -0.2131 & 0.512 \\
\hline ISBP mean*cerebral perfusion status & -0.0599 & 0.123 & - & - & - & - \\
\hline Centered ISBP mean & & & -0.0470 & 0.01 & -0.0918 & 0.018 \\
\hline Quadratic term of mean & & & 0.0009 & 0.355 & 0.0009 & 0.38 \\
\hline $\begin{array}{l}\text { Centered ISBP mean*cerebral } \\
\text { perfusion status }\end{array}$ & & & - & - & -0.0538 & 0.157 \\
\hline Age & & & & & & \\
\hline Preoperative SBP mean & -0.0280 & 0.052 & -0.0288 & 0.046 & -0.0281 & 0.053 \\
\hline Operation time & 0.0149 & 0.003 & 0.0439 & 0.003 & 0.0414 & 0.006 \\
\hline Operation site & -0.00126 & 0.484 & -0.0012 & 0.505 & -0.0012 & 0.502 \\
\hline Previous stroke & 0.3907 & 0.022 & 0.3809 & 0.025 & 0.3849 & 0.024 \\
\hline Pre-operative Hemoglobin & 0.0450 & 0.855 & 0.0787 & 0.749 & 0.0589 & 0.812 \\
\hline Post-operative Hemoglobin & 0.3866 & 0.005 & 0.3805 & 0.006 & 0.3730 & 0.007 \\
\hline Length of hospital stay & -0.2010 & 0.156 & -0.1746 & 0.219 & -0.1774 & 0.213 \\
\hline SBP, Systolic Blood Pressure & 0.1089 & 0.002 & 0.0966 & 0.004 & 0.1021 & 0.004 \\
\hline Reference group: bad cerebral perfusion status, right site of operation, no previous stroke & \\
\hline
\end{tabular}

The proportions of model-predicted TND cases per mean ISBP categories were plotted per mean ISBP, whereby average and reference values were fixed for continuous and categorical variables, respectively. While the proportion of TND cases in patients with a good cerebral perfusion status declined alongside the mean ISBP, the proportion of TND cases in patients with a bad cerebral perfusion status declined dramatically as the mean ISBP increased (Fig. 2).

\section{Discussion}

This study has shown that low ISBP was independently associated with the risk of postoperative TND, following STA-MCA anastomosis in adult patients with MMD. In patients with a poor perfusion status, 
low ISBP further increased the risk of TND.

The association between intraoperative SBP and risk of stroke has been reported in patients undergoing cardiac[14] or general surgery.[15] These previous studies emphasized the importance of preventing intraoperative hypotension for maintaining cerebral perfusion during surgery. Consistent with these studies, our findings suggest that the risk of TND after revascularization surgery for MMD might be associated with low ISBP, which might cause local intraoperative hypoperfusion. A recent study has demonstrated that local hypoperfusion measured with cerebral blood flow was correlated with transient neurologic events.[16] A variety of factors affect low blood pressure during surgery, including anaesthetics. The anaesthetic agents commonly used for neurosurgery, such as propofol and remifentanil, can lower blood pressure. In the present study, phenylephrine was used to maintain BP above the preoperative baseline value. Although the effect of phenylephrine has not been studied in patients with MMD, administration vasopressors to patients with ischemic stroke has been associated with improvement in neurologic outcomes.[17]

In the present study, cerebrovascular reserve was associated with the risk of TND. Poor preoperative perfusion status increased the risk of TND when ISBP decreased. Most ischemic events in patients with MMD have been ascribed to reduced blood flow caused by the narrowing of cerebral arteries.[18] Previously, an examination with postoperative perfusion SPECT imaging has revealed that patients with a decreased cerebrovascular reserve are at risk of neurologic deficits and ischemic attacks.[11] The present study emphasizes the role of perfusion status alongside changes to blood pressure in managing these outcomes.

Operation to the left side is a well-documented risk factor for hyperperfusion after STA-MCA anastomosis.[7, 19] Similarly, operation to the left side was associated with the risk of TND in the present study. These findings suggest that surgery to the left side requires careful management, including maintenance of adequate cerebral flow during surgery, which might require more fine-tuning compared to when operating on the right side.

The present study has some limitations. First, selection bias was inevitable due to the retrospective nature of this study. Second, our data analysis was performed per hemisphere rather than per patient, meaning we were not able to account for cases of patients who had undergone surgery on both sides (right and left). We were not able to discount the possibility that double surgery might affect outcomes. Third, factors related to the type of surgery (e.g. direct or combined bypass) were not accounted for in the analysis. Lastly, we did not consider postoperative BP as a confounder in this study, which might limit the validity of our results. Although BP was strictly controlled during the postoperative period, based on the present findings, low postoperative BP cannot be excluded as a risk factor for TND.

\section{Conclusions}

In summary, low ISBP might be associated with TND risk, following STA-MCA anastomosis in adult patients with MMD. In patients with poor perfusion status, low ISBP increased the risk of TND. Our 
findings suggest that strict ISBP control might be required to prevent TND in this patient group, in particular, in patients with a poor perfusion status.

\section{Abbreviation List}

ISBP, Intraoperative systolic blood pressure; TND, transient neurologic deficits; STA-MCA, superficial temporal-to-middle cerebral artery; MMD, moyamoya disease; $C T$, computed tomography; SPECT, singlephoton emission computed tomography; CVR, cerebrovascular reserve; $\mathrm{OR}$, odds ratio; $\mathrm{Cl}$, confidence interval

\section{Declarations}

\section{Ethics approval and consent to participate}

This retrospective observational study was approved by the institutional review board of Seoul National University Bundang Hospital (IRB No.B-1411/274-113). The requirement for patient consent was waived due to the retrospective nature of this study and minimal risk to study participants.

\section{Consent for publication}

not applicable

\section{Competing interests}

The authors declare that they have no competing interests

\section{Funding}

None

\section{Authors' contributions}

Tak Kyu Oh: The author designed study, drafted paper, helped data acquisition, and data analysis, and approved the final paper.;Ji Hyeon Kim, Ho-Young Lee,Seong-Eun Kim, Tackeun Kim,Beom Joon Kim,Jae Seung Bang,Moon-Ku Han,Chang Wan Oh: The authors helped data acquisition, data interpretation, and critical revisions of paper, and approved the final paper;Hee-Joon Bae andYoung-Tae Jeon: The authors designed study, drafted paper, helped data acquisition, and data analysis, and approved the final paper.;

\section{Acknowledgments}

The authors thank the Medical Informatics team at Seoul National University Bundang Hospital for their dedicated work.

\section{Availability of data and materials}


The datasets used and/or analysed during the current study available from the corresponding author on reasonable request.

\section{References}

1. Kim T, Oh CW, Kwon OK, Hwang G, Kim JE, Kang HS, Cho WS, Bang JS. Stroke prevention by direct revascularization for patients with adult-onset moyamoya disease presenting with ischemia. $J$ Neurosurg. 2016;124(6):1788-93.

2. Fujimura M, Kaneta T, Mugikura S, Shimizu H, Tominaga T. Temporary neurologic deterioration due to cerebral hyperperfusion after superficial temporal artery-middle cerebral artery anastomosis in patients with adult-onset moyamoya disease. Surgical neurology. 2007;67(3):273-82.

3. Kim JE, Oh CW, Kwon OK, Park SQ, Kim SE, Kim YK. Transient hyperperfusion after superficial temporal artery/middle cerebral artery bypass surgery as a possible cause of postoperative transient neurological deterioration. Cerebrovasc Dis. 2008;25(6):580-6.

4. Ohue S, Kumon Y, Kohno K, Watanabe H, Iwata S, Ohnishi T. Postoperative temporary neurological deficits in adults with moyamoya disease. Surgical neurology. 2008;69(3):281-6. discussion 286287.

5. Hyun SJ, Kim JS, Hong SC. Prognostic factors associated with perioperative ischemic complications in adult-onset moyamoya disease. Acta Neurochir. 2010;152(7):1181-8.

6. Fujimura M, Mugikura S, Kaneta T, Shimizu H, Tominaga T. Incidence and risk factors for symptomatic cerebral hyperperfusion after superficial temporal artery-middle cerebral artery anastomosis in patients with moyamoya disease. Surgical neurology. 2009;71(4):442-7.

7. Hwang JW, Yang HM, Lee H, Lee HK, Jeon YT, Kim JE, Lim YJ, Park HP. Predictive factors of symptomatic cerebral hyperperfusion after superficial temporal artery-middle cerebral artery anastomosis in adult patients with moyamoya disease. Br J Anaesth. 2013;110(5):773-9.

8. Chung JW, Kim N, Kang J, Park SH, Kim WJ, Ko Y, Park JH, Lee JS, Lee J, Yang MH, et al. Blood pressure variability and the development of early neurological deterioration following acute ischemic stroke. J Hypertens. 2015;33(10):2099-106.

9. Parray T, Martin TW, Siddiqui S. Moyamoya disease: a review of the disease and anesthetic management. J Neurosurg Anesthesiol. 2011;23(2):100-9.

10. Iwama T, Hashimoto N, Yonekawa Y. The relevance of hemodynamic factors to perioperative ischemic complications in childhood moyamoya disease. Neurosurgery. 1996;38(6):1120-5. discussion 1125-1126.

11. So Y, Lee HY, Kim SK, Lee JS, Wang KC, Cho BK, Kang E, Lee DS. Prediction of the clinical outcome of pediatric moyamoya disease with postoperative basal/acetazolamide stress brain perfusion SPECT after revascularization surgery. Stroke. 2005;36(7):1485-9.

12. Kim T, Oh CW, Bang JS, Kim JE, Cho WS. Moyamoya Disease: Treatment and Outcomes. J Stroke. 2016;18(1):21-30. 
13. Kim JM, Lee SH, Roh JK. Changing ischaemic lesion patterns in adult moyamoya disease. J Neurol Neurosurg Psychiatry. 2009;80(1):36-40.

14. Sun LY, Chung AM, Farkouh ME, van Diepen S, Weinberger J, Bourke M, Ruel M. Defining an Intraoperative Hypotension Threshold in Association with Stroke in Cardiac Surgery. Anesthesiology. 2018;129(3):440-7.

15. Bijker JB, Persoon S, Peelen LM, Moons KG, Kalkman CJ, Kappelle LJ, van Klei WA. Intraoperative hypotension and perioperative ischemic stroke after general surgery: a nested case-control study. Anesthesiology. 2012;116(3):658-64.

16. Mukerji N, Cook DJ, Steinberg GK. Is local hypoperfusion the reason for transient neurological deficits after STA-MCA bypass for moyamoya disease? J Neurosurg. 2015;122(1):90-4.

17. Hillis AE, Ulatowski JA, Barker PB, Torbey M, Ziai W, Beauchamp NJ, Oh S, Wityk RJ. A pilot randomized trial of induced blood pressure elevation: effects on function and focal perfusion in acute and subacute stroke. Cerebrovascular diseases. 2003;16(3):236-46.

18. Kim DY, Son JP, Yeon JY, Kim GM, Kim JS, Hong SC, Bang OY. Infarct Pattern and Collateral Status in Adult Moyamoya Disease: A Multimodal Magnetic Resonance Imaging Study. Stroke. 2017;48(1):111-6.

19. Fujimura M, Niizuma K, Inoue T, Sato K, Endo H, Shimizu H, Tominaga T. Minocycline prevents focal neurological deterioration due to cerebral hyperperfusion after extracranial-intracranial bypass for moyamoya disease. Neurosurgery. 2014;74(2):163-70. discussion 170.

\section{Figures}




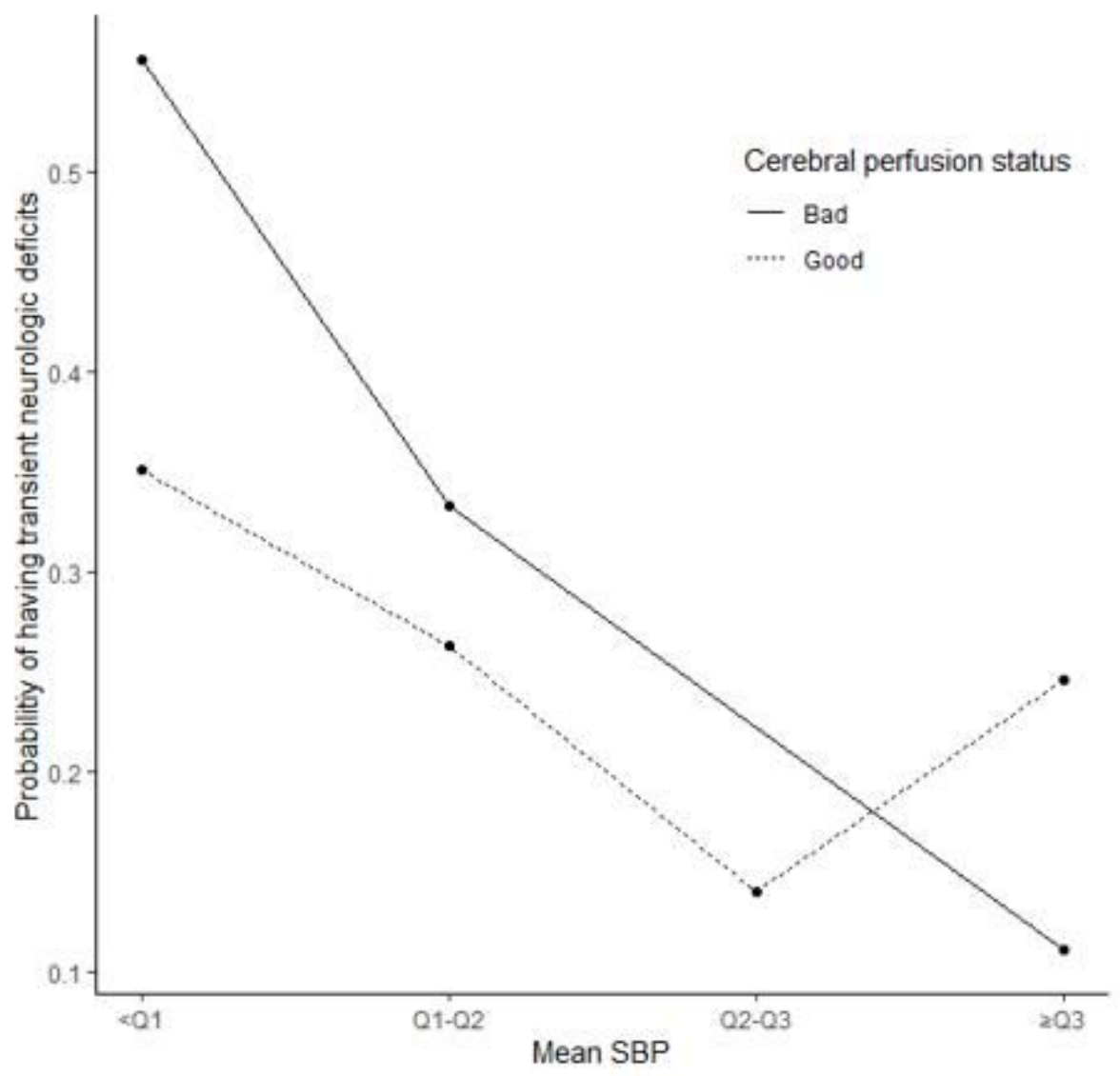

Figure 1

Proportion of transient neurologic deficits across quartile group of mean systolic blood pressure (SBP) 


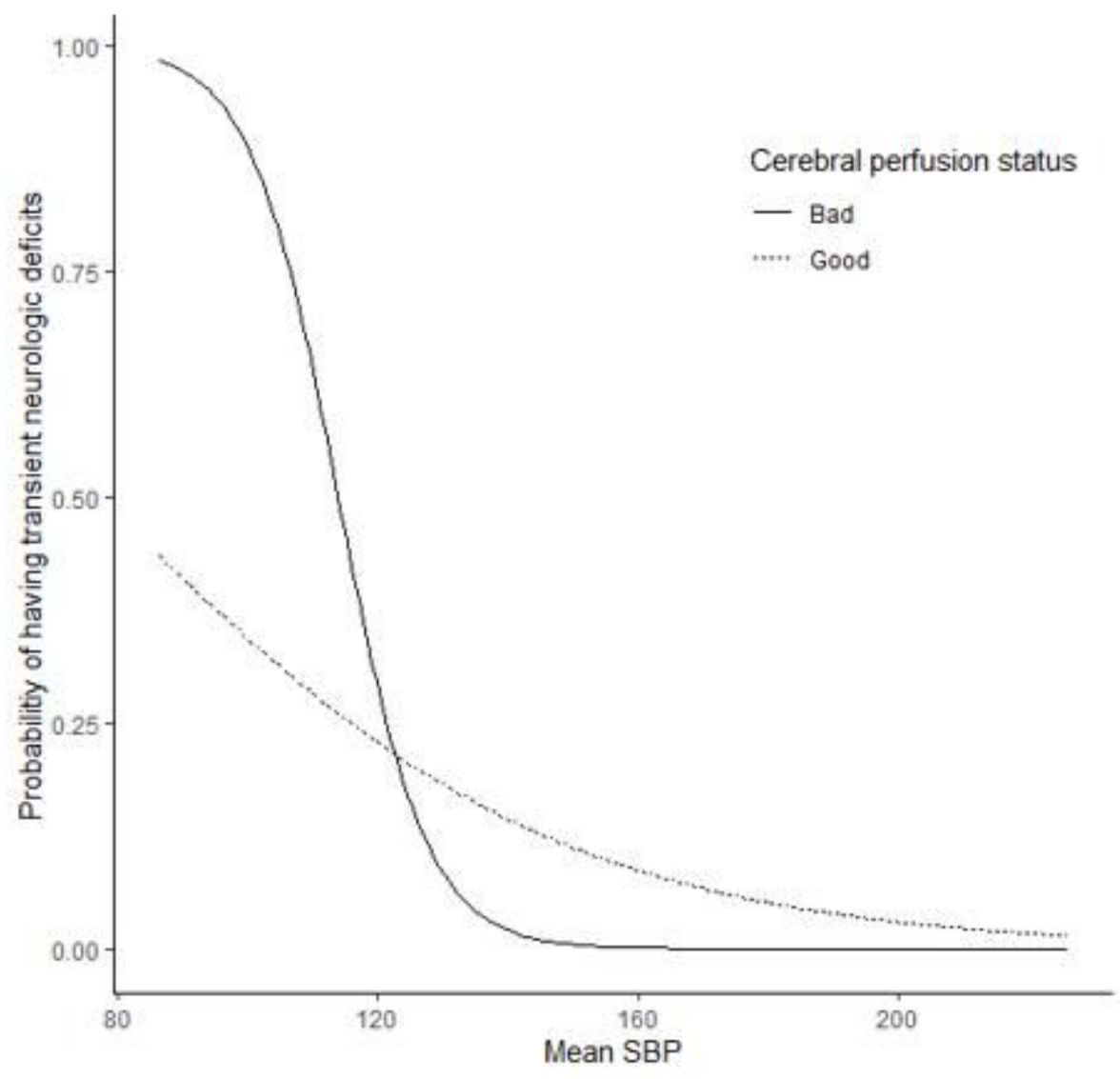

Figure 2

Predicted probabilities of transient neurologic deficits derived from a linear logistic regression model on mean systolic blood pressure (SBP) 